Estrategias de mercadeo social dirigidas a incentivar la inclusión de personas con discapacidad en el sector farmacéutico No. 2 | Volumen 1 | Abril - Junio 2017 http://revistaenfoques.org ISSN: 2616 - 8219

\section{ESTRATEGIAS DE MERCADEO SOCIAL DIRIGIDAS A INCENTIVAR LA INCLUSIÓN DE PERSONAS CON DISCAPACIDAD EN EL SECTOR FARMACÉUTICO}

\author{
SOCIAL MARKETING STRATEGIES AIMED AT ENCOURAGING THE \\ INCLUSION OF PEOPLE WITH DISABILITIES IN THE PHARMACEUTICAL \\ SECTOR
}

Gabriela Linares A.

\section{Resumen}

Las empresas en Venezuela han empezado a dar valor con la puesta en práctica de las estrategias de mercadeo dirigidas al compromiso solidario, a fin de favorecer el bienestar común de la sociedad. Por ende, el propósito de estudio fue crear estrategias de mercadeo social orientadas a la incorporación de las personas con discapacidad. La investigación se llevó cabo mediante un estudio factible centrado en la construcción de estrategias de mercadeo social, fue tipo descriptiva-explicativa, bajo el diseño de campo, se combinaron técnicas de la encuesta y documentales. Los resultados resaltantes revelan que los factores que influyen en el diseño e implementación de estrategias de mercadeo social para la incorporación de las personas con discapacidad son de carácter subjetivo la desconfianza que genera por el impacto negativo que pueda tener tanto en la productividad $2,92 \%$ y el clima laboral $2,83 \%$ de la compañía, así como, el desconocimiento de las leyes relacionadas con contratación de personal discapacitado $2,83 \%$. En cuanto a los factores de tipo objetivo obedecen a elementos financieros.

Palabras clave: Mercadeo social; plan de mercadeo social, discapacidad

\begin{abstract}
Companies in Venezuela have begun to give value with the implementation of marketing strategies aimed at solidarity commitment, in order to promote the common welfare of society. Therefore, the purpose of the study was to create social marketing strategies aimed at incorporating people with disabilities. The research was carried out through a feasible study focused on the construction of social marketing strategies, it was descriptive-explanatory type, under the field design, survey techniques and documentaries were combined. The outstanding results reveal that the factors that influence the design and implementation of social marketing strategies for the incorporation of people with disabilities are of a subjective nature the distrust generated by the negative impact that may have on both productivity $2.92 \%$ and the company's $2.83 \%$ working environment, as well as, ignorance of the laws related to hiring disabled personnel $2.83 \%$. As for the objective type factors, they are due to financial elements.
\end{abstract}

Key words: Social marketing; social marketing plan; disabilities

\section{Gabriela Linares A.}

mchmechizarza@yahoo.es mchmechizarza@hotmail.com Universidad Central de Venezuela

Doctoranda en Gestión de la Investigación y Desarrollo en la Universidad Central de Venezuela 2019, recibida como Especialista en Gerencia de Proyectos en la Universidad Católica Andrés Bello en el año 2009, realizó un Diplomado en Gestión de Gobierno Electrónico en la Universidad Dr. José Gregorio Hernández en el año 2014, y Egresada como Licenciada en Ciencias Estadísticas en la Universidad Central de Venezuela en el año 2006. Ha realizado actividades vinculadas a la Ciencia, Tecnología e Innovación a lo largo de 14 años en donde los proyectos más relevantes en su trayectoria laboral ha sido la Formulación y Aplicación de Indicadores de Ciencia y Tecnología en el Marco del ALBA-TCP, Diseño Metodológico para la Gestión de Proyectos de la UCAB enmarcados en las directrices de la LOCTI, Participación en la realización del boletín de Indicadores Venezolanos de Ciencia, Tecnología e Innovación, así como el cumplimiento con instituciones nacionales e internacionales 


\section{INTRODUCCIÓN}

En las últimas décadas se ha observado como las empresas en Venezuela han empezado a dar valor a la puesta en práctica de las estrategias de mercadeo dirigidas al compromiso solidario de favorecer del bienestar común de la sociedad. Una de estas estrategias es el mercadeo social por parte del sector empresarial dirigido a la identificación de las necesidades, deseos y/o preferencias de los consumidores y a orientar a toda la organización hacia la satisfacción de estas necesidades sin descuidar sus objetivos económicos y financieros.

Cuando las empresas hacen un esfuerzo estratégico para apoyar la solución a problemas sociales fortalecen su imagen ante la sociedad y motivan la lealtad de sus empleados y consumidores. En este sentido, las estrategias de mercadeo social pueden ser útiles a las empresas, no solo para responder a las exigencias del entorno en el tema social sino, también para fortalecer su imagen como empresas socialmente responsables. Una de las claves para alcanzar los objetivos de las organizaciones consiste en ser más eficaz que la competencia, además, de ofrecer un mayor valor al público, por lo que es importante entender que la necesidad y satisfacer las necesidades del consumidor.

De esta realidad no escapa la industria farmacéutica venezolana, en la cual existe la inquietud de diseñar estrategias de mercadeo social aplicado a este sector de la económica el cual permitirá conocer más a fondo cada uno de los factores que lo integran y a su vez, aplicar las herramientas necesarias para su implementación con el propósito de alcanzar el mejoramiento de la calidad de vida.
Estas estrategias de mercadeo social están orientadas a la incorporación de las personas con discapacidad, para ello, fue necesario llevar a cabo un estudio centrado en desarrollar estrategias de mercadeo social que incentiven el empleo de personas con discapacidad en el campo laboral dentro de algunas empresas del sector farmacéutico, a fin de aumentar las oportunidades laborales de los ciudadanos con algún padecimiento, al mismo tiempo que se fortalece la imagen de la industria ante la sociedad como empresas de la diversidad.

En la actualidad, es cada vez más complejo el posicionamiento de las empresas en el mercado, tanto nacional como mundial, producto entre otros factores del proceso de globalización y los grandes cambios generados por los avances tecnológicos, el desarrollo profesional de los diferentes equipos de talento humano y las constantes transformaciones de los intereses, gustos, necesidades y requerimientos de los clientes meta.

Este esquema de crecimiento económico debe igualmente, promover el establecimiento de estrategias que contribuyan con la generación de mejoras sustanciales en la calidad de vida de la población. Kotler (2010) plantea que, a partir de la década de 1970, algunos de los académicos en la Escuela de Gerencia de Kellogg de la Universidad de, Northwestern introdujeron la idea que, el marketing se puede aplicar a una gama más amplia de temas más allá de la comercialización de bienes y servicios.

En la actualidad el componente social del mercadeo plantea que, las empresas coexisten con un contenido ético orientado a la utilidad que puedan prestar a la sociedad. Por tanto, el gran reto del mercadeo es alcanzar que las empresas generen 
beneficios económicos y la vez sean socialmente responsables como beneficiosas para la sociedad.

El diseño de estas estrategias se concibió como "mercadeo social" y su objetivo principal, más allá de proponer acciones aisladas de responsabilidad social, pretende proponer acciones que generen beneficios laborales sostenibles en el tiempo a través de un proceso de integración, la eliminación de estereotipos personales, laborales que contribuyan al desarrollo económico y social de la sociedad. El marketing social tiene como finalidad el bienestar de la población objetivo y, de ser posible, de la sociedad en general de acuerdo con algunos autores como, Kotler y Armstrong (2003).

El diseño, implantación y control de programas que buscan incrementar la aceptabilidad de una idea social o prácticas en grupos. Para ello, se aplican los conceptos de segmentación de mercados, investigación de mercado, comunicación, facilidad, incentivos y se genera el cambio de utilidad por la satisfacción máxima del grupo. (Kotler y Armstrong (2003).

Este tipo de comercialización podría ayudar a crear interés en los lugares, personas, organizaciones, ideas y causas. La competencia dejo de ser un concepto exclusivo del mercado de bienes de consumo e industriales por lo que, se aplica de igual modo para que otros sectores como el social o público. Este conjunto de herramientas se dirige a vender mejores comportamientos, bajo este esquema de mercadeo social las empresas incentivan un cambio de conducta, el precio que puede ser el propio cambio de conducta, el tiempo o incluso dinero en el cambio de conducta, la distribución que consiste en tener los elementos necesarios para que el individuo cambie de conducta.
En este contexto, la aplicación del mercadeo social constituye una excelente estrategia para el cambio de conducta en la percepción sobre la incorporación de personas con algún tipo de discapacidad en el mercado laboral, ya que, en la actualidad, un gran número de personas con discapacidad desean trabajar, pero enfrentan diversos obstáculos que le impiden insertarse en el campo laboral.

En Venezuela existen pocas experiencias de mercadeo social dirigidas a la incorporación en el sector laboral de las personas con discapacidad PCD de acuerdo con cifras del Consejo Nacional para la Integración de Personas con Discapacidad (CONAPDIS) el registro de personas con discapacidad laboralmente activas para la fecha era de apenas 25785, este número de personas incorporadas obedece al cumplimiento de la Ley de Personas con Discapacidad (LPCD), la cual establece la obligatoriedad tanto de los entes públicos como privados contar en nómina con un porcentaje mínimo del $5 \%$ de personas con discapacidad y no responde a la puesta en práctica de un plan de mercadeo social.

Entre las experiencias existentes de mercadeo social destaca la implementada por la industria farmacéutica especialmente, la empresa Laboratorio GlaxoSmithKline en la cual la Junta Directiva aprobó la elaboración y puesta en práctica de un proyecto para la inclusión de PCD, adaptado a la empresa, su personal y a las necesidades de este grupo. La propuesta se convirtió en un piloto que fue evaluado, ajustado e implementado por la empresa y que servirá como base para la propuesta de las estrategias de marketing social que fomenten la contratación asertiva de PCD basadas en las capacidades y aptitudes reales de cada una de estas personas de 
forma que respondan a las expectativas de las funciones que desempeñan.

En vista de ello, se hace necesario el diseño de las estrategias de mercadeo social que, puestas en práctica de manera sistemática incorporen programas de mercadeo social enmarcados dentro de la gestión de responsabilidad social corporativa aseguren la sustentabilidad de las mismas generando así beneficios tanto para las personas con discapacidad como para las empresas socialmente responsables.

En relación a los antes expuesto, surgió la iniciativa de diseñar estrategias de mercadeo social que incentiven la contratación de personas con discapacidad en la industria farmacéutica, pertenecientes a la globalización y la competitividad de los productos y servicios. Lo cual ha obligado a las empresas a estar preparados a las exigencias del mercado, a fin para garantizar y asegurar el éxito en el posicionamiento del mercado. Para ello, fue necesario hacer uso de técnicas y herramientas para conocer las exigencias y necesidades de los clientes meta.

Desde hace algunos años las organizaciones se han incentivado con la implementación de estrategia de mercadeo social, bajo un esquema de rentabilidad y generación de beneficios económicos para promover la calidad de vida de estas personas, mediante la generación de cambios, actitudes y transformaciones en la práctica, para ello son incorporados dentro del proceso de mercadeo, a través del vínculo que les promociona la marca de un producto o servicio con causas sociales en las cuales se identifica y se compromete con dicha transformación.

Al implementar estrategias de mercadeo social en la industria farmacéutica venezolana, las empresas de este ramo de la economía tienen la posibilidad de incentivar en sus clientes potenciales los cambios de actitudes hacia las personas con discapacidad. De igual manera, se espera que con ello, se incremente el posicionamiento en el mercado de marca e imagen y esta se refleje en la rentabilidad del sector.

Con el diseño de estrategias de mercadeo social dirigidas a incentivar la inclusión de personas con discapacidad se espera un mayor nivel de sensibilización hacia el cambio de conductas nocivas no solo para este sector poblacional, sino para el bien común, promoviendo con ello la generación de nuevas iniciativas públicas y privadas.

Las estrategias de mercadeo social representan un instrumento útil que sirve de guía a todas las personas involucradas con las actividades de mercadeo de la empresa, ya que describe las formas y actividades de cómo se debe desarrollar y aplicar los mecanismos de control y monitoreo, no solo para el posicionamiento de la empresa en el mercado, sino también para impulsar los cambios de actitudes, la promoción de nuevas ideas aventajando la identificación de la empresa para de esta manera estimular la transformación social.

La importancia que encontró este estudio radica en el diseño de estrategias desde la perspectiva de mercadeo social, dirigidas a lograr la comunicación y la motivación de los clientes meta, para transfórmalas en herramientas que permitan posicionar la imagen que se persigue de la empresa con relación al aspecto social. Además, la investigación contribuyó a dar un aporte en la realización de planes de mercadeo social.

Estas estrategias de mercadeo social contribuirán con las personas con discapacidad para promocionar sus facultades, habilidades y destrezas en el 
desempeño en el sector laboral. Para la empresa, representa una estrategia que le garantizará el cumplimiento de la responsabilidad social empresarial (RSE) y una forma original para incentivar a otras empresas a la contratación de trabajadores con discapacidad. Adicionalmente, el presente estudio contribuyó como un valioso aporte al estudio del mercadeo social como herramienta para favorecer la inclusión social de grupos con necesidades especiales, constituyéndose en una fuente de consulta para futuras investigaciones relativas al tema.

\section{MÉTODOS}

La investigación consistió en el diseño de estrategia de mercadeo social dirigidas a incentivar la incorporación de personas con discapacidad en las empresas transnacionales de la Industria Farmacéutica pertenecientes a CAVEME, ésta se llevó a cabo en la ciudad de Caracas, Venezuela. La modalidad fue mediante el proyecto factible, de tipo descriptivo-explicativo bajo el diseño de campo. Se aplicó como técnica la encuesta usando como instrumento el cuestionario para la recogida de la información. Además las preguntas estuvieron orientadas con a determinar la problemática diagnostica y el análisis de los datos permitió el diseño de estrategias que permitieran a la organización objeto de estudio crear actividades para mejorar su imagen con relación a la labor social.

RESULTADOS Y DISCUSIÓN

A continuación se detallan los resultados arrojados durante la elaboración de este estudio, lo que perimirá conocer un poco más acerca de la estrategia que fue creada para incrementar la labor social en la industria farmacéutica venezolana, ésta estuvo orientada a inducir a un conjunto de empresas a incorporarse al programa, el cual se realizó mediante el desarrollo de un producto inicial, una marca para el producto y una estrategia promocional, sobre la base de las necesidades iníciales que surgieron del análisis de los resultados mediante la encuetas realizada al público meta potencial.

\section{Inclusión de personas con discapacidad}

Las categorías de respuesta de esta pregunta fueron: SI y NO ante lo cual el $53 \%$ de los encentados respondieron negativamente que no poseen personal con discapacidad. Por otra parte, el $66 \%$ de los encuestados respondieron negativamente que no es parte de un programa tal y como puede apreciarse en el Tabla 1. Inclusión de Personas con Discapacidad.

Tabla 1. Inclusión de Personas con Discapacidad

\begin{tabular}{lccccc}
\hline Opciones & Si & \% & NO & $\%$ & Cantidad de respuestas \\
\hline Responsabilidad social & 8 & 53,33 & 7 & 46,67 & 15 \\
Mercadeo social & 6 & 40,00 & 9 & 60 & 15 \\
Inclusión del personal & 7 & 46,67 & 8 & 53,33 & 15 \\
No es parte de un programa & 5 & 33,33 & 10 & 66,67 & 15 \\
\hline
\end{tabular}




\section{Empresa sin empleados con discapacidad}

El comportamiento de las respuestas de los encuestados puede observarse en la tabla 2, en donde se destacan las razones en las cuales la sector farmacéutico actualmente no hay empleados con discapacidad.

Tabla 2. Razones por las cuales en su empresa actualmente no hay empleados con discapacidad

\begin{tabular}{|c|c|c|c|c|c|}
\hline & $\begin{array}{c}\text { De } \\
\text { acuerdo }\end{array}$ & Indiferente & $\begin{array}{c}\text { En } \\
\text { desacierdo }\end{array}$ & Total & $\begin{array}{l}\text { Promedio } \\
\text { ponderado }\end{array}$ \\
\hline No se tiene un programa establecido & $41,67 \%$ & $8,33 \%$ & $50,00 \%$ & 12 & 2,08 \\
\hline $\begin{array}{l}\text { relacionado con la contratacion del } \\
\text { personal con discapacidad }\end{array}$ & 5 & 1 & 6 & & \\
\hline Problemas de presupuesto para & $53,85 \%$ & --- & $56,15 \%$ & 13 & 1,92 \\
\hline $\begin{array}{l}\text { hacer contratacion de este tipo de } \\
\text { personal }\end{array}$ & 7 & & 6 & & \\
\hline Dificil situacion financiera de la & $66,67 \%$ & $8,33 \%$ & $25,00 \%$ & 12 & 2,42 \\
\hline compañía & 8 & 1 은 & 3 & & \\
\hline Desconocimiento de como & $25,00 \%$ & $8,33 \%$ & $66,67 \%$ & 12 & 2,42 \\
\hline $\begin{array}{l}\text { implementar programas de } \\
\text { mercadeo social- responsabilidad } \\
\text { social que permitan la contratacion } \\
\text { de personal con discapacidad }\end{array}$ & 3 & 1 은 & 8 & & \\
\hline Desconocimiento de las leyes & $8,33 \%$ & $8.33 \%$ & $83,33 \%$ & 12 & 2,75 \\
\hline relacionadas con la contratación & 1 & 1 & 10 & & \\
\hline Posibilidad de impactar & --- & $16,67 \%$ & $83,33 \%$ & 12 & 2,83 \\
\hline $\begin{array}{l}\text { negativamente la productividad de } \\
\text { la compañía }\end{array}$ & & 2 & 10 & & \\
\hline Posibilidad de impactar & --- & $8,33 \%$ & $91,67 \%$ & 12 & 2,92 \\
\hline $\begin{array}{l}\text { negativamente el clima laboral de la } \\
\text { compañía al momento de contratar } \\
\text { personal con discapacidad }\end{array}$ & & 1 & 11 & & \\
\hline \multirow[t]{2}{*}{ Ausencia de posiciones vacantes } & $50,00 \%$ & --- & $50,00 \%$ & 12 & 2,00 \\
\hline & 6 & & 6 & & \\
\hline Ausencia de posiciones vacantes con & $53,85 \%$ & $7,69 \%$ & $38,46 \%$ & 13 & 1,85 \\
\hline $\begin{array}{l}\text { perfil que pueda ser asumido por } \\
\text { una persona con discapacidad }\end{array}$ & 7 & 1 & 5 & & \\
\hline Falta de claridad de los beneficios & $33,33 \%$ & --- & $66,67 \%$ & 12 & 2,33 \\
\hline $\begin{array}{l}\text { para la compañía a contratar } \\
\text { personal con discapacidad }\end{array}$ & 4 & & 8 & & \\
\hline
\end{tabular}


De acuerdo con la tendencia de las personas encuestadas en cuanto a los factores que influyen en el diseño e implementación de estrategias de mercadeo social para la incorporación de las personas con discapacidad, el comportamiento fue que un alto porcentaje como puede apreciar en la tabla 2 esta en desacuerdo que las razones sean de tipo subjetivo como la desconfianza que genera por el impacto negativo que pueda tener tanto en la productividad y el desconocimiento de las leyes relacionadas con contratación de personal discapacitado.

Por otra parte, la tendencia de los encuestados sobre los factores por los cuales no contrante personal con discapacida son factores de tipo objetivo, existe una marcada tendencia un alto porcentaje de los encuestados como puede apreciar en la tabla 2 esta de acuerdo que las razones sean de tipo subjetivo como problemas de presupuesto para hacer contratación de éste tipo de personal, la difícil Situación Financiera de la compañía y la ausencia de posiciones vacantes con un perfil que pueda ser asumido por una persona con discapacidad.

La segunda parte de la pregunta abordó la prioridad asignada a las 3 razones principales por las que en la empresa no hay empleados con Discapacidad, según una escala donde 1 es la más relevante o prioritaria, 2 medianamente relevante y 3 la menos relevante.

También, como se puede apreciar el gráfico 1 , la tendencia de las respuestas de los encuestados se observa que la opción "Posibilidad de Impactar negativamente el clima laboral de la compañía al momento de contratar personal discapacitado". La segunda es la "Posibilidad de Impactar negativamente la productividad de la compañía" ambas opciones responden a factores de tipo subjetivo, sin embargo, este escenario puede transformarse, ya que al incorporar una persona con discapacidad a un lugar de trabajo, los empleadores pueden obtener asesoramiento y orientación recurriendo a los servicios de organismos especializados sobre cómo implementar programas de Mercadeo Social y Responsabilidad social que permita la contratación de personal con discapacidad. Ante lo cual, se hace evidente la necesidad de una campaña promocional de sensibilización y concientización para el cambio de percepción que algunas compañías tienen acerca de la incorporación a puestos de trabajo de personas con discapacidad.

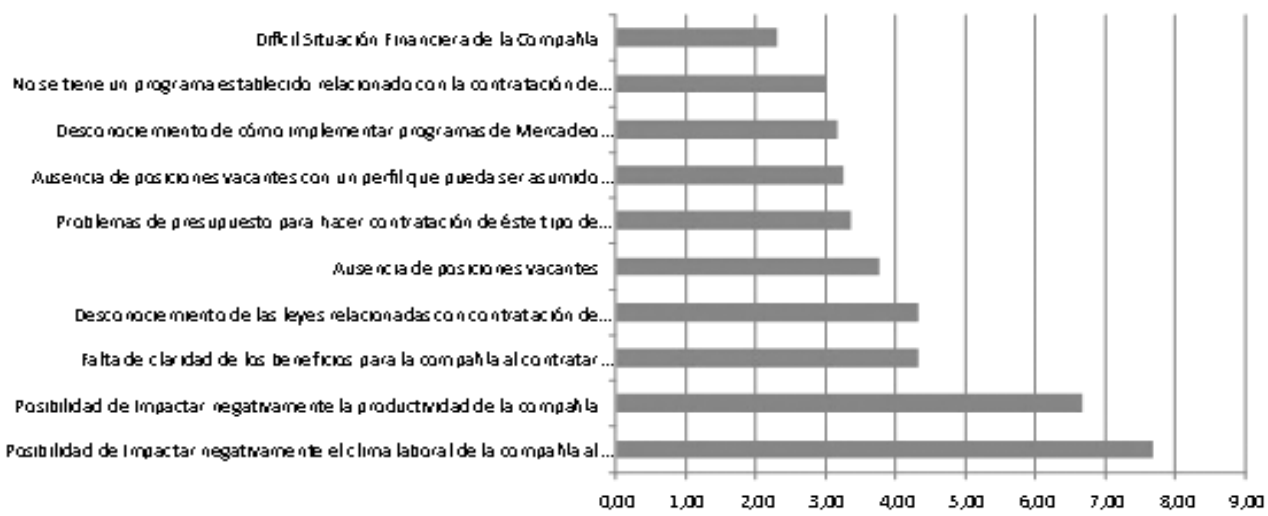

Gráfico 1. Principales por las que en la empresa no hay empleados con discapacidad 
Los componentes de las estrategias de mercadeo social que respondan a las necesidades de la empresa y estén alineados a la normativa legal y las políticas nacionales deben ser diseñados considerando los factores que inciden en la negativa para la integración laboral de las personas con discapacidad. A efectos de Marketing, tenemos un producto que es el mercadeo social que posee dos clientes: uno externo, el empresario y otro interno el aspirante a trabajador, así como un eje de comunicación que consiste en reiterar que el término "Discapacidad" no es sinónimo de "incapacidad laboral". En este orden de ideas, la muestra seleccionada considera que la opción puede representar una solución para dar inicio a la contratación de este talento humano, lo cual puede apreciarse el gráfico 2 .

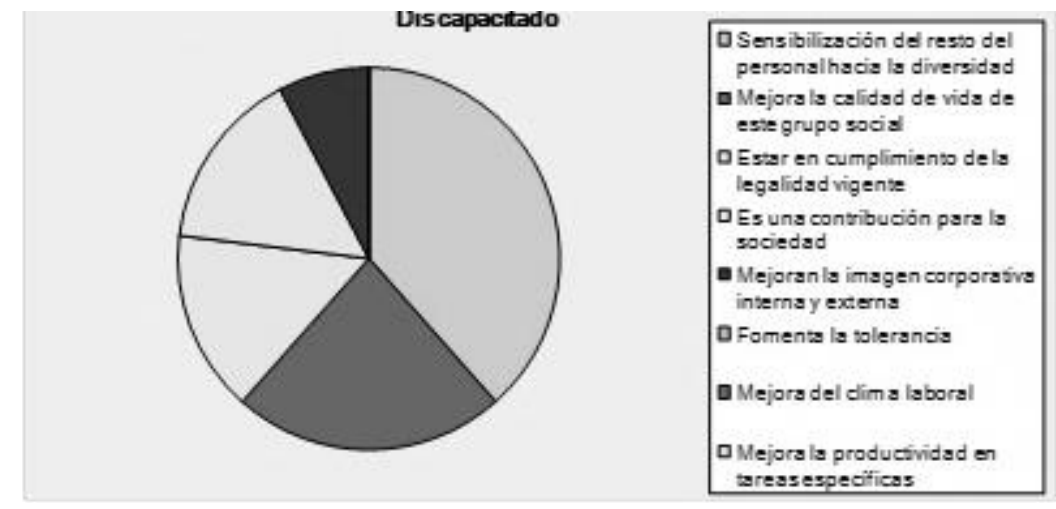

Gráfico 2. Beneficios a la compañía por la contratación de personal con discapacidad

Entre las opciones planteadas por la muestra que faciliten la integración de las personas con discapacidad al sector laboral, destacan la identificación de tareas que pueden ser realizadas por una persona con discapacidad, contar con una base de datos de perfiles de personas con discapacidad, obtención de un beneficio fiscal por la contratación del $5 \%$ establecido en la ley, contar con un presupuesto que permita crear un programa de mercadeo social o de responsabilidad social, flexibilización de la ley de personas con discapacidad.

La aplicación de las estrategias de mercadeo social que respondan a un doble motivo por una parte, las necesidades de la empresa y por otra, que estén alineados a la normativa legal y las políticas nacionales que regula este tipo de problemática, la aplicación del marketing social podrían contribuir de forma activa a mejorar los niveles de integración de las personas con discapacidad y a las empresas al cumplimiento de la norma y la eliminación de estereotipos, personales y laborales, sobre estos ciudadanos.

Es así como, se observa en el gráfico 3 la sensibilización del resto del personal es la principal opción que contribuirá a la contratación de este tipo de talento humano, dado el grado de desconocimiento en algunos representantes de las empresas sobre el tema de la integración laboral de las personas con discapacidad en general, los ciudadanos no están sensibilizados ante esta problemática. 


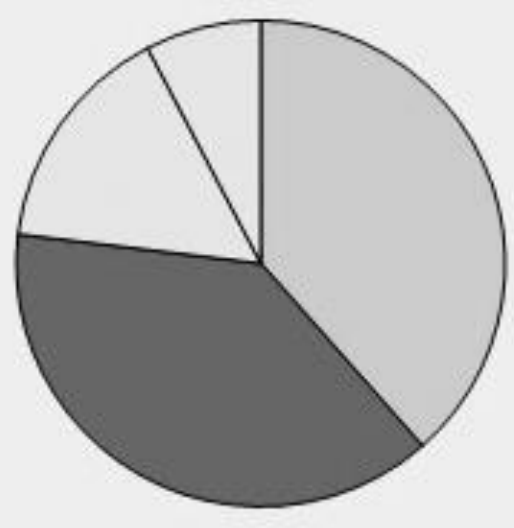

口Nohay Empleados

Discapacitadosactualmente

laborando en la empresa

- Menosde 3 Empleados con

Discapacidad

口 Entre 3 a 5 Empleados con

Discapacidad

口 Más5 Empleados con

Discapacidad

Grafico 3. Cantidad de personas con algún tipo de discapacidad que actualmente laboran en la compañía

La sensibilización constituye una herramienta fundamental de la estrategia para la promoción del empleo de personas con discapacidad, cuando se analiza el gráfico 3 se observa que el $38 \%$ de las empresas encuestadas no posee empleados con discapacidad y el otro $38 \%$ sólo posee menos de tres empleados, datos que permiten inferir que el $77 \%$ de las empresas apenas si contratan menos de tres empleados con discapacidad.
Aunado a lo anterior, el poco porcentaje de personas con discapacidad solo es contratado por periodos de tiempo cortos lo cual puede evidenciarse en el gráfico 4 el cual refleja, de acuerdo con la información suministrada por la muestra seleccionada, que el tiempo promedio que permanece un empleado con discapacidad en su empresa es de cinco (5) años.

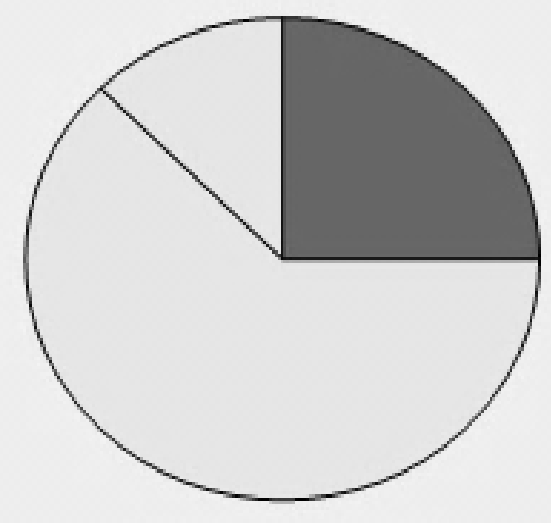

- Menosde 1 año

$\square$ De 1 a 3 años

$\square$ De 3 a 5 años

๑ Más de 5 años

Gráfico 4. Tiempo promedio (años) que permanece un empleado con discapacidad en su empresa en la compañía 
Ante lo anteriormente planteado, la sensibilización contiene un componente informativo que fomenta actitudes positivas y solidarías fundamentadas en el derecho de las personas con discapacidad y el respeto a la diversidad, convivencia con personas con discapacidad. Mediante la sensibilización, las empresas adquieren conciencia de las limitaciones y a la vez, aprenden a valorar las capacidades, aspectos fundamentales para iniciar el conocimiento.

En cuanto a la incorporación por tipo de discapacidad se puede apreciar al analizar el gráfico 4, que el mayor número de personas con discapacidad contratadas son las que tiene como condición la discapacidad física. Ante ello, el objetivo de la sensibilización consiste en brindar herramientas $y$ conocimientos que permitan comprender las potencialidades y limitaciones de quienes tienen esta condición, instruir sobre otros tipos de discapacidad y patologías, mejorar la comunicación del potencial de estas personas, fomentar su integración laboral y divulgar información con miras a prevenir los accidentes que conllevan a esta situación en los casos que aplique.

Como se mencionó en párrafos anteriores, entre los factores por los cuales no se contrata a personas con discapacidad es porque se tiene la percepción de que dicha contratación no representa ventajas ni para la empresa ni para el trabajador. La situación del empleo, y particularmente la inserción laboral, ha tenido escaso desarrollo en el mercadeo social. Es por ello, que las estrategias sociales propuestas por esta investigación adicionalmente al tipo de discapacidad a la que deben estar orientadas, tienen como objetivo sensibilizar a las empresas a la incorporación en condición de igual de género al igual que del resto de sus potencialidades, habilidades y sobre la vinculación con el mercado de trabajo

Cuando se observa los datos que arroja el Gráfico 3 se evidencia que el mayor porcentaje de personas contratadas en condición de discapacidad pertenece al género femenino.

En función de los resultados obtenidos y analizados en el capítulo anterior seguidamente se presenta la propuesta de estrategias de marketing social orientadas a la creación de oportunidades para la incorporación de las personas con discapacidad en el sector farmacéutico, dando cumplimiento al tercer objetivo específico de la investigación.

\section{Estrategias de mercadeo social dirigidas a incentivar la inclusión de personas con discapacidad en el sector farmacéutico}

La realización e implementación de las estrategias de mercadeo social permitirán la creación de oportunidades para la incorporación de las personas con discapacidad en el sector farmacéutico. La estrategia de mercadeo social a implementarse en las principales empresas transnacionales de la industria farmacéutica. El marketing social es un proceso para crear, comunicar y entregar los beneficios que un público (s) objetivo quiere a cambio de comportamiento del público que beneficia a la sociedad sin beneficio económico para el vendedor.

\section{Objetivo}

Motivar a las empresas transnacionales de la industria farmacéutica pertenecientes a CAVEME a considerar como parte de los procesos de selección y captación del talento humano la incorporación de las personas con discapacidad en el sector como acciones solidarias que, sin implicar una inversión 
importante de tiempo o recursos, están destinados a prestar algún tipo de ayuda al otro.

\section{Producto social}

Desarrollo de estrategias de mercadeo social que fomenten la contratación asertiva de personas con discapacidad basadas en las capacidades y aptitudes reales de cada una de estas personas de forma que respondan a las expectativas de las funciones que desempeñan, como herramienta para alcanzar que las empresas generen beneficios económicos y la vez sean socialmente responsables como beneficiosas para la sociedad.

\section{Enfoque}

Incorporación laboral de las personas con discapacidad en el sector farmacéutico.

\section{Causa}

Incorporar en el mercado laboral a las personas con discapacidad.

\section{Destinatarios}

Como parte de la fase de planificación, específicamente de la segmentación de audiencia, se determinó como público meta las principales empresas transnacionales de la industria farmacéutica pertenecientes a CAVEME. Según los datos arrojados posterior a la aplicación de los instrumentos de recolección de datos, el público meta tiene una noción básica del comportamiento que se quiere promover, sin embargo, desconocen sus implicaciones legales a profundidad sobre la importancia de la contratación del personal con discapacidad y en ellos, priva la percepción negativa sobre el impacto en la productividad y el clima organizacional. Por ende, es importante que, como parte de la primera etapa de esta estrategia, se diseñen mensajes destinados a proporcionar al público meta mensajes dirigidos a modificar esta percepción.

\section{Cambio de comportamiento deseado}

El proceso de investigación permitió identificar elementos importantes en cuanto a las actitudes del público meta, los cuales son considerados en el diseño de la propuesta, ya que a través de estos no sólo se construirán mensajes que le sean afines al público meta, sino que se definió la forma en que estos les serán transmitidos o difundidos. Partiendo de los diferentes resultados previos al diseño de esta herramienta, es posible establecer las siguientes actitudes hacia la incorporación de las personas con discapacidad en el sector laboral: Generar confianza en las empresas farmacéuticas sobre la importancia de la contratación de personal discapacitado modificando la percepción sobre el impacto negativo que pueda tener tanto en la productividad y el clima laboral de la compañía así como, así como en el desconocimiento de las leyes relacionadas.

\section{Entorno}

Las estrategias a desarrollar serán a través de las redes sociales como Facebook, Twitter, Mensajería de Texto, Whatsapp. Sin embargo, en la actualidad el entorno donde se desarrollan está caracterizado por el momento crítico que vive el país marcado por la agudización de las dificultades económicas, el desempleo, la inseguridad personal, la dificultad en las divisas de las empresas del sector farmacéutico venezolano.

\section{Mensaje}

Se pretende transmitir mensajes a la alta gerencia de empresas transnacionales de la 
industria farmacéutica pertenecientes a CAVEME, en relación a los conceptos, definiciones y características de las personas con discapacidad, modelos de empleo, programas de transición laboral, así como el análisis y alcances de la legislación y las áreas potencialmente factibles para la integración. Los puntos principales que se desean transmitir en este mensaje son:

- Soy una persona con habilidades diferentes

- ¡Enséñales a los demás a integrarlos!

- Valorar las diferencias significativas

- Crear un ambiente donde las diferencias no dividan a las personas, sino que contribuyan a un alto desempeño, experiencias en el trabajo y de calidad de vida más interesantes.

- Nuestras diferencias representan distintas maneras de pensar, sentir, expresarnos, vivir y ser. Esto incluye diferencias de estilos personales, estilos de trabajo, actitud mental y estilos de comunicación.

- Un entorno en el que las diferencias se valoran se fomenta el respeto y la confianza en cada persona.

\section{Canales de comunicación del mensaje}

- Individuo u organización que intenta generar un cambio social Individuos: la empresa Laboratorio GlaxoSmithKline.

- Grupos o poblaciones enteras que son objetivo de los llamados al cambio: las principales empresas transnacionales de la industria farmacéutica pertenecientes a CAVEME.

- Vías de comunicación y distribución, a lo largo de las cuales se intercambian y transmiten hacia atrás y adelante la influencia y respuesta entre los agentes de cambio se utilizarán las redes sociales como:

- Facebook: se utiliza fundamentalmente para transmitir fotografías y videos, saber cuál es el comportamiento de los seguidores y hacer pequeñas encuestas para conocer los cambios de comportamientos

- Twitter: Esta red social es utilizada fundamentalmente para transmitir mensajes dirigidos al cambio de modificación en la actitud de las empresas transnacionales de la industria farmacéutica pertenecientes a CAVEME.

- Correo Electrónico: Esta red social es utilizada fundamentalmente para difundir información para orientar. Esta red social será utilizada fundamentalmente para difundir información para orientar las empresas transnacionales de la industria farmacéutica pertenecientes a CAVEME sobre las descripciones de cargos y alinearlas a las competencias socio laborales de las personas con discapacidad, estudiar la accesibilidad en las áreas de trabajo previamente identificadas, las tareas y actividades asociadas a esos roles a fin de garantizar que efectivamente pueden ser desempeñadas por este personal.

Páginas Web: Esta red social es utilizada fundamentalmente para difundir información para orientar las empresas transnacionales de la industria farmacéutica pertenecientes a CAVEME sobre las descripciones de cargos y alinearlas a las competencias socio laborales de las personas con discapacidad, estudiar la accesibilidad en las áreas de trabajo previamente identificadas, las tareas y actividades asociadas a esos roles a fin de garantizar que efectivamente pueden ser desempeñadas por este personal. 


\section{Segmento}

La segmentación de audiencia, se determinó en función del público meta conformado por las principales empresas transnacionales de la industria farmacéutica pertenecientes a CAVEME. Este público meta constituido por:

- Segmento Marca Representa el 72\% del mercado total, manteniendo el nivel de crecimiento en el orden del $4 \%$ al cierre del 2010.

- Segmento Genérico Mantiene su participación sobre el mercado total en $28 \%$, presentando un crecimiento en unidades en $11 \%$.

- Segmento Ético Presenta para el 2010 un crecimiento del $6 \%$.

- Segmento OTC Presenta un crecimiento del 6\% al 2010, con él y una participación sobre el total mercado del $33 \%$

\section{Posicionamiento}

El posicionamiento en el mercado será como líderes, ya que es la primera experiencia en lanzar este tipo de servicio por parte de la industria farmacéutica, es una estrategia innovadora de marketing social $y$ se implementara mediante tecnología avanzada en la empresa.

\section{Estrategia}

La propuesta fue diseñada en función de los pasos señalados por Kotler y Lee (2008), para los cuales el marketing social representa el cambio de comportamiento a través de la aplicación de las campañas y los esfuerzos para influir en la acción social.

\section{Producto}

Incorporación de las personas con discapacidad en el mercado laboral del sector farmacéutico mediante la generación de confianza en las empresas farmacéuticas sobre la importancia de la contratación de personal discapacitado modificando la percepción sobre el impacto negativo que pueda tener tanto en la productividad y el clima laboral de la compañía, así, como en el desconocimiento de las leyes relacionadas.

\section{Precio}

En el mercado social el precio va íntimamente ligado a la sensación de calidad del producto (así como a su exclusividad). Sin embargo, es necesario estimar los principales costos de implementación de la estrategia como es necesario para desarrollo contar especialmente con un especialista en mercadeo social, no obstante, dada la situación país y los niveles de inflación existentes es difícil establecer el costo real.

\section{Plaza}

En este caso se define como dónde comercializar el producto o el servicio que se le ofrece (elemento imprescindible para que el producto sea accesible para el consumidor). El mercadeo social se encarga de implementar ideas sociales que mejoren alguna actitud negativa, pero se debe conocer en qué lugar se va a ofrecer este nuevo producto para que sea exitoso. Para Kotler (2003), la plaza comprende las actividades de la empresa que ponen al producto a disposición de los consumidores meta. En el caso del plan de mercadeo social para la incorporación laboral de las personas con discapacidad en el sector farmacéutico, este se desarrollará en el área metropolitana de Caracas, mediante las redes sociales y los medios digitales.

\section{Promoción}

Como bien se mencionó en el Capítulo II la promoción consiste en comunicar, 
informar y persuadir al cliente y otros interesados sobre la empresa, sus productos y ofertas, para el logro de los objetivos organizacionales. La mezcla de promoción está constituida por promoción de ventas, fuerza de venta o venta personal, publicidad y relaciones públicas, y comunicación interactiva. La promoción consiste según lo explica Kotler (2003) en dar a conocer, informar, recordar, educar, persuadir y concienciar a la población cuyo objetivo o mercado meta acerca de los productos y servicios de las organizaciones sin fines de lucro en busca del bienestar social.

El canal de comunicación para la promoción se realizará mediante las redes sociales. A través de los cuales se quiere comunicar que las personas con discapacidad son personas con Habilidades Diferentes, el mensajero de la promoción es la empresa Laboratorio GlaxoSmithKline.

\section{CONCLUSIONES}

En la actualidad el componente social del mercadeo plantea que las empresas coexisten con un contenido ético orientado a la utilidad que puedan prestar a la sociedad. Por tanto, el gran reto del mercadeo es alcanzar que las empresas generen beneficios económicos y la vez sean socialmente responsables como beneficiosas para la sociedad.

El mercadeo social tiene como propósito ayudar a crear interés en los lugares, personas, organizaciones, ideas y causas donde la competencia dejo de ser un concepto exclusivo del mercado de bienes de consumo e industriales, este conjunto de herramientas se dirige a vender mejores comportamientos, bajo este esquema de mercadeo social las empresas incentivan un cambio de conducta, el precio que puede ser el propio cambio de conducta, el tiempo o incluso dinero en el cambio de conducta, la distribución que consiste en tener los elementos necesarios para que el individuo cambie de conducta.

En Venezuela existen pocas experiencias de mercadeo social dirigidas a la incorporación en el sector laboral de las personas con discapacidad PCD, entre las experiencias existentes de mercadeo social destaca la implementada por la industria farmacéutica especialmente, la empresa Laboratorio GlaxoSmithKline en la cual la Junta Directiva aprobó la elaboración y puesta en práctica de un proyecto para la inclusión de PCD, adaptado a la empresa, su personal y a las necesidades de este grupo. La propuesta se convirtió en un piloto que fue evaluado, ajustado e implementado por la empresa y que serbio como base para la propuesta de las estrategias de marketing social que fomenten la contratación asertiva de PCD basadas en las capacidades y aptitudes reales de cada una de estas personas de forma que respondan a las expectativas de las funciones que desempeñan.

Se llevó a cabo el diagnóstico para evaluar las razones por las cuales no son incorporadas las personas con discapacidad al sector farmacéutico, para ello se realizó una encuesta a la muestra seleccionada, la cual arrojó como resultado las razones por las cuales no son incorporadas las personas con discapacidad al sector farmacéutico. Estas razones se pueden categorizar en dos conjuntos de opciones para establecer los factores que influyen en el diseño e implementación de estrategias de mercadeo social para la incorporación de las personas con discapacidad.

El primer conjunto de factores son de tipo subjetivo, el principal factor que incide en la falta de contratación de personas con 
discapacidad en la desconfianza que genera por el impacto negativo que pueda tener tanto en la productividad $2,92 \%$ y el clima laboral 2,83\% de la compañía así como, el desconocimiento de las leyes relacionadas con contratación de personal discapacitado $2,83 \%$.

El segundo conjunto de factores que limitan la contratación de personas con discapacidad obedece a elementos objetivo de tipo financieros asociados a la situación que inciden en el comportamiento de la selección del recurso humano de cualquier empresa la ausencia de una No se tiene un programa establecido relacionado con la contratación de personal entre ellas el $2,08 \%$, señalo que la ausencia de un programa establecido relacionado con la contratación de personal discapacitado, el $2,00 \%$ la ausencia de posiciones vacantes, el $1,92 \%$ problemas de presupuesto para hacer contratación de éste tipo de personal, el $1,85 \%$ la ausencia de posiciones vacantes con un perfil que pueda ser asumido por una persona con discapacidad y, el 1,58\% la difícil situación financiera de la compañía.

Si se analizó desde el enfoque de Marketing, los factores que influyen en la incorporación de las personas con discapacidad, lo cual arrojó como conclusión que son principalmente, tres, el entorno especialmente el económico y el social frente a lo cual los encuestados respondieron como una de las causas la situación país y Difícil Situación Financiera de la Compañía. El segundo elemento, el cliente, tanto el externo como el interno entorno a lo que la muestra seleccionada plantea que existe una percepción de la posibilidad de Impactar negativamente el clima laboral de la compañía al momento de contratar personal discapacitado, y, por último, el mercado o la competencia elemento que incide significativamente ya que, uno de las razones por las cuales no está integrada esta población es por la posibilidad de impactar negativamente la productividad de la compañía.

Con el diseño de estrategias de mercadeo social dirigidas a incentivar la inclusión de personas con discapacidad se espera un mayor nivel de sensibilización hacia el cambio de conductas nocivas no solo para este sector poblacional sino para el bien común, promoviendo con ello la generación de nuevas iniciativas tanto públicas como privadas.

Los componentes de la estrategia que, de acuerdo con los resultados obtenidos deben estar orientados a definir los objetivos del programa para el cambio social, son la causa, los destinatarios, los canales, el producto social que los agentes de cambio consideran que ofrecerá una respuesta acertada a un problema social, el individuo u organización que intenta generar un cambio social Individuos, los grupos o poblaciones enteras que son objetivo de los llamados al cambio, las vías de comunicación y distribución, a lo largo de las cuales se intercambian y transmiten hacia atrás y adelante la influencia y respuesta entre los agentes de cambio, la dirección y el programa adoptados por un agente de cambio, para llevar a cabo el cambio en las actitudes y conducta de los destinatarios.

Tal como estaba previsto, en el plan propuesto se estableció como objetivo motivar a las empresas transnacionales de la industria farmacéutica pertenecientes a CAVEME a considerar como parte de los procesos de selección y captación del talento humano la incorporación de las personas con discapacidad en el sector como acciones solidarias que, sin implicar una inversión importante de tiempo o recursos, están destinados a prestar algún tipo de ayuda al otro. 
En cuanto a, la estrategia está orientada a generar confianza en las empresas farmacéuticas sobre la importancia de la contratación de personal discapacitado modificando la percepción sobre el impacto negativo que pueda tener tanto en la productividad y el clima laboral de la compañía, así como, así como en el desconocimiento de las leyes relacionadas.

En cuanto a la estrategia comunicacional, se plantearon los siguientes mensajes Soy una Persona con Habilidades Diferentes, Enséñales a los demás a integrarlos, Valorar las diferencias significa crear un ambiente donde las diferencias no dividan a las personas, sino que contribuyan a un alto desempeño y a experiencias de trabajo y de vida más interesantes, Nuestras diferencias representan distintas maneras de pensar, sentir, expresarnos, vivir y ser. Esto incluye diferencias de estilos personales, estilos de trabajo, actitud mental y estilos de comunicación, un entorno en el que las diferencias se valoran se fomenta el respeto y la confianza en cada persona.
Las estrategias generales del plan, se enfocaron a fomentar la contratación asertiva de personas con discapacidad basadas en las capacidades y aptitudes reales de cada una de estas personas de forma que respondan a las expectativas de las funciones que desempeñan, como herramienta para alcanzar que las empresas generen beneficios económicos y la vez sean socialmente responsables como beneficiosas para la sociedad, mediante el diseño mensajes destinados a proporcionar al público meta mensajes dirigidos a modificar esta percepción.

\section{REFERENCIAS}

Kotler, P., y Armstrong, G. (2003). Fundamentos de marketing. Pearson Educación.

Kotler, P. (2010). El movimiento prosumidor. En Prosumer Revisited (pp. 51-60). VS Verlag für Sozialwissenschaften

Kotler, P., y Lee, N. (2008). Social marketing: Influencing behaviors for good. Sage.

Kotler, P. (2003). Dirección de marketing: conceptos esenciales. Pearson educación. 